\title{
Ice-Templated MXene/Ag-Epoxy Nanocomposites as High-Performance Thermal Management Materials
}

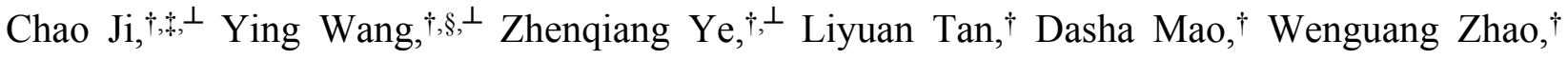 \\ Xiaoliang Zeng, ${ }^{\dagger}$ Changzeng Yan, ${ }^{\dagger}, *$ Rong Sun, ${ }^{\dagger}, *$ Dae Joon Kang,,$\|, *$ Jianbin Xu, ${ }^{*}$ and Ching- \\ Ping Wong $\mathrm{T}$ \\ $\dagger$ Shenzhen Institute of Advanced Electronic Materials - Shenzhen Fundamental Research \\ Institutions, Shenzhen Institutes of Advanced Technology, Chinese Academy of Sciences, \\ Shenzhen 518055, China \\ $\$$ College of Materials Science and Engineering, Shenzhen University, Shenzhen 518055, China \\ $\S$ College of Chemical Engineering and Environment, China University of Petroleum, Beijing \\ 102249, China \\ || Department of Physics, Sungkyunkwan University, 2066, Seobu-ro, Jangan-gu, Suwon, \\ Gyeonggi-do 16419, Republic of Korea \\ = Department of Electronics Engineering, The Chinese University of Hong Kong, Hong Kong, \\ China \\ T School of Materials Science and Engineering, Georgia Institute of Technology, Atlanta, \\ Georgia 30332, United States \\ *E-mail: cz.yan@siat.ac.cn; rong.sun@siat.ac.cn; djkang@skku.edu. \\ ${ }^{\perp}$ Chao Ji, Ying Wang, and Dr. Xiaoliang Zeng contributed to this work equally.
}




\section{Table of Contents:}

S1. SEM image of the purchased MAX

S2. High-resolution C1s XPS spectra of MXene and MXene/Ag

S3. The measurement method of electrical conductivity and Electrical conductivities of MXene/Ag films with different Ag loadings

S4. Calculation of ITR between fillers and the epoxy resin matrix

S5. Thermal conductivities vs. Ag particle loading (particle loading refers to the weight ratio of Ag NPs to MXene sheets)

S6. The image of ice template method application system

S7. infrared thermal images of epoxy, MXene-epoxy and MXene/Ag-epoxy nanocomposites after different heating times. 


\section{S1. SEM image of the purchased MAX}

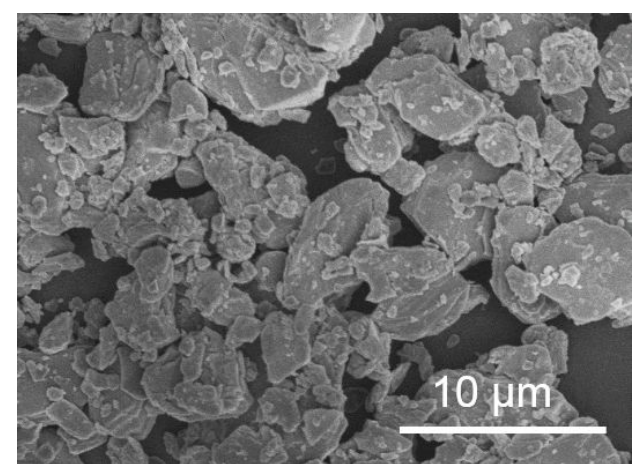

Figure S1. SEM image of the purchased MAX.

\section{S2. High-resolution C1s XPS spectra of MXene and MXene/Ag}

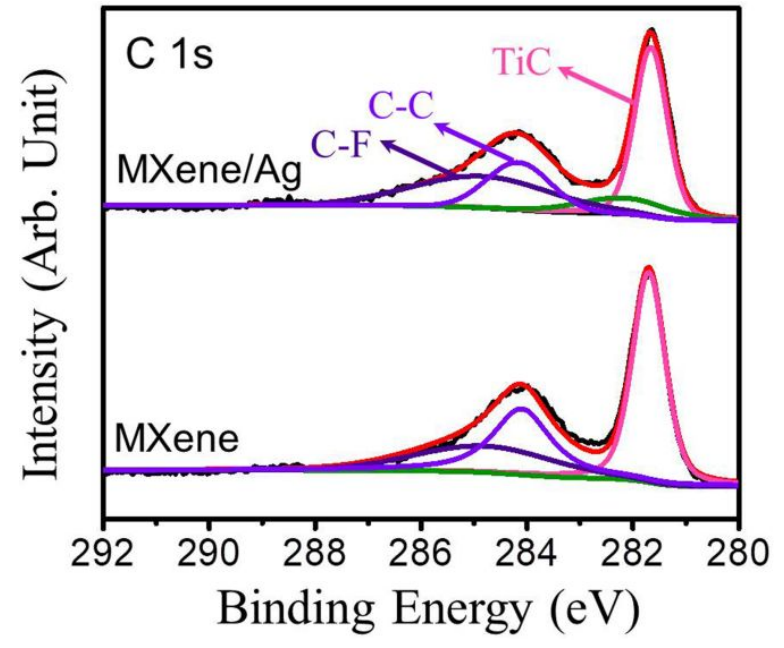

Figure S2. High-resolution C1s XPS spectra of MXene and MXene/Ag.

S3. The measurement method of electrical conductivity and Electrical conductivities of

\section{MXene/Ag films with different Ag loadings}

Here we use the resistivity tester (Japan EC-80P) to measure the square resistance of the film. According to the following formulas:

$$
\mathrm{R}=\rho / \mathrm{d}
$$




$$
\sigma=1 / \rho
$$

The formula for calculating the conductivity is obtained:

$$
\sigma=1 / R d
$$

Where $\mathrm{R}$ is the square resistance, ohm/sqr, $\mathrm{d}$ is the thickness, $m$, and $\sigma$ is the electrical conductivity, $S / m$. respectively.

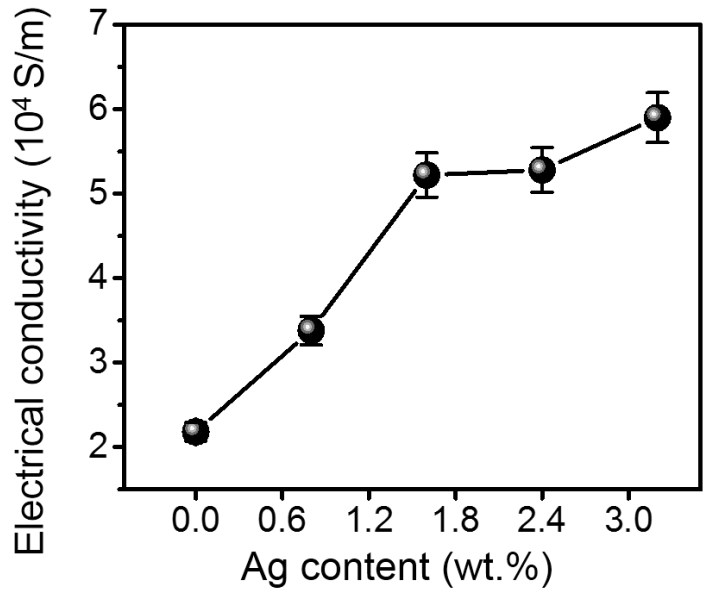

Figure S3. Electrical conductivities of MXenes/Ag films with different Ag loadings.

\section{S4. Calculation of ITR between fillers and the epoxy resin matrix}

In this experiment, the thermal conductivity of MXenes with a size of $3 \mu \mathrm{m}$ and diameter of $2 \mathrm{~nm}$ (aspect ratio $\alpha$ is $c a$. 1500) was evaluated.

$$
V_{c}(\alpha \gg 1)=\frac{0.6}{\alpha}
$$

Critical fractional volume $\left(V_{c}\right)$ was determined to be $0.04 \%$ from Equation S2. According to previous reports, ${ }^{\mathrm{S} 1}$ when $\alpha=0.0004, t(\alpha)$ can be calculated as 1.3 .

$$
\mathrm{K}\left(V_{f}, \alpha\right)=K_{0}\left(\mathrm{~V}_{f}-\mathrm{V}_{c}\right)^{t(\alpha)}
$$

Therefore, $K_{0}=30.6-45.8 \mathrm{~W} \mathrm{~m}^{-1} \mathrm{~K}^{-1}$.

$$
R_{c}=\left(K_{0} L V_{c}^{t(\alpha)}\right)^{-1}
$$

It can be calculated that $R_{\mathrm{c} / \mathrm{MXenes} / \mathrm{Ag}}=2.8-2.9 \times 10^{8} \mathrm{~K} \mathrm{~W}^{-1}$.

Similarly, for MXene-epoxy composites, $R_{\mathrm{c} / \mathrm{MXenes}}=3-3.5 \times 10^{7} \mathrm{~K} \mathrm{~W}^{-1}$. 


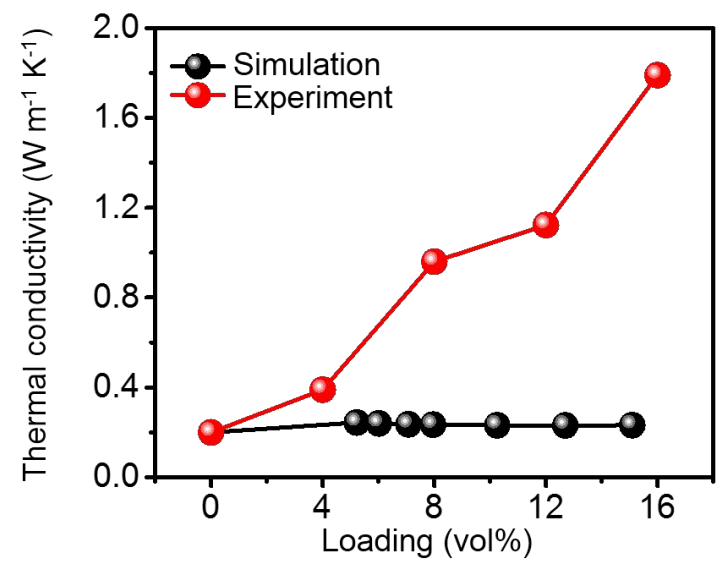

Figure S4. Simulated and experimental in-plane thermal conductivities of MXene/Ag-epoxy nanocomposites at different filler loadings.

\section{S5. Thermal conductivity $v$ s. Ag particle loading (particle loading refers to the weight ratio of Ag NPs to MXene sheets)}

The simulated in-plane thermal conductivity showed a slight decrease when the filler (sheet and particle) loading increased. As discussed earlier in the main text, the large increase in inplane thermal conductivity may be attributed to the non-flat nature and interaction of sheets; further, Ag NPs increase interactions between individual MXene sheets.

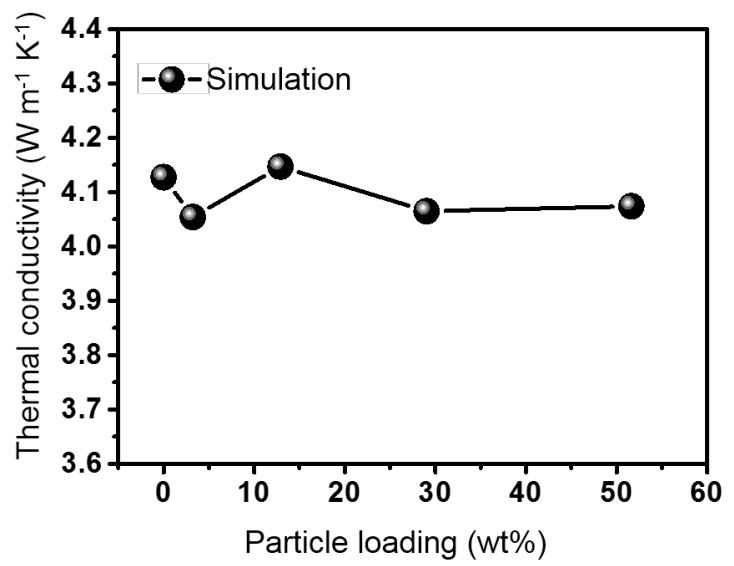

Figure S5. Thermal conductivity of MXene/Ag-epoxy nanocomposites as a function of $\mathrm{Ag}$ particle loading.

No significant improvement was found according to simulation since the MXene sheets and Ag NPs were uniformly distributed in the epoxy matrix. Increasing the amount of silver particles can enhance the interaction between sheets, hence improvement can be expected when sheets are not ideally flat. 
No significant improvement could be observed in the simulation results as MXene sheets and Ag NPs were uniformly distributed in the epoxy matrix. Increasing the loading amount of silver particles can enhance interactions between MXene sheets and hence an improvement in thermal conductivity can be expected when sheets are not ideally flat.

\section{S6. The image of ice template method application system}
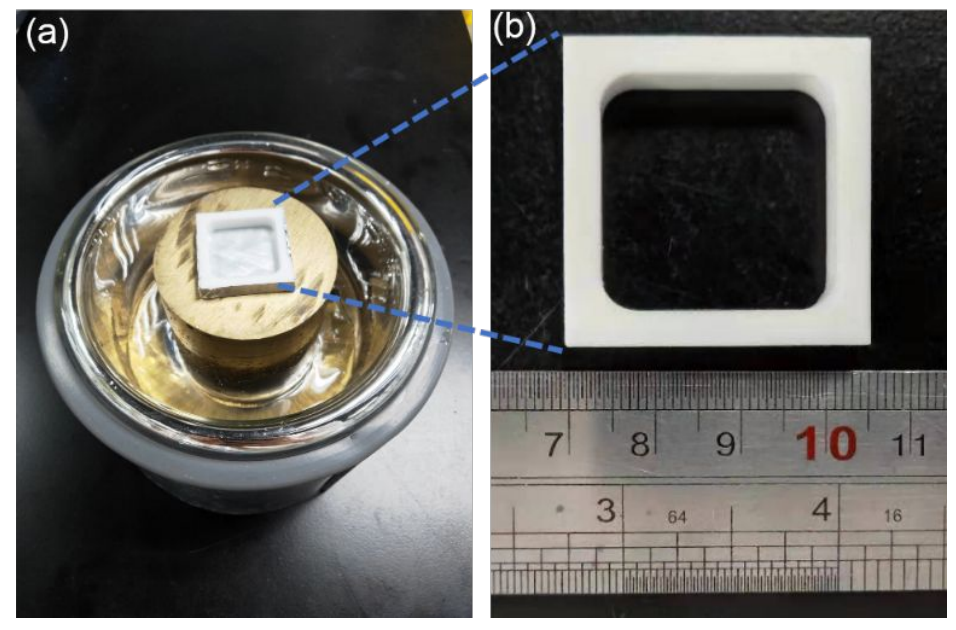

Figure S6. (a) Ice template method application system, (b) the mold of ice template in (a) and its size

\section{S7. infrared thermal images of epoxy, MXene-epoxy and MXene/Ag-epoxy} nanocomposites after different heating times.

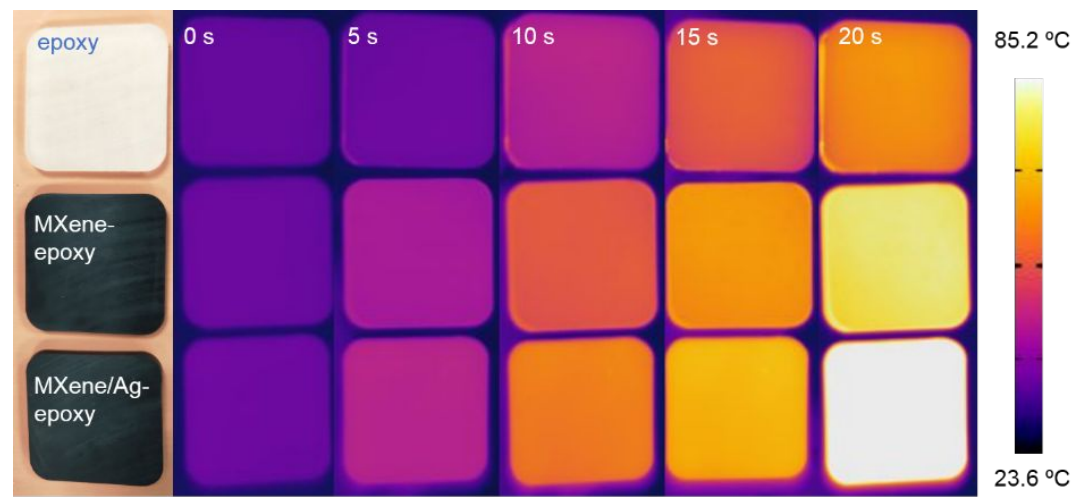

Figure S7. infrared thermal images of epoxy, MXene-epoxy and MXene/Ag-epoxy nanocomposites after different heating times.

\section{Reference}


S1. Sengupta, I.; Udgaonkar, J. B., Structural mechanisms of oligomer and amyloid fibril formation by the prion protein. Chem. Commun. 2018, 54 (49), 6230-6242. 